\title{
An Evolutionary Algorithm for Graph Planarisation by Vertex Deletion
}

\author{
Rodrigo Lankaites Pinheiro ${ }^{1}$, Ademir Aparecido Constantino ${ }^{2}$, Candido F. X. de Mendonça ${ }^{3}$ \\ and Dario Landa-Silva ${ }^{1}$ \\ ${ }^{1}$ School of Computer Science, University of Nottingham, NG8 1BB, Nottingham, U.K. \\ ${ }^{2}$ Informatics Department, State University of Maringá, Maringá, PR, Brazil \\ ${ }^{3}$ School of Arts, Science and Humanities, USP-East, São Paulo, SP, Brazil \\ psxrp2@nottingham.ac.uk,aaconstantino@uem.br, cfxavier@usp.br,dario.landasilva@nottingham.ac.uk
}

Keywords: Graph Planarisation, Evolutionary Algorithms, Vertex Deletion.

\begin{abstract}
A non-planar graph can only be planarised if it is structurally modified. This work presents a new heuristic algorithm that uses vertices deletion to modify a non-planar graph in order to obtain a planar subgraph. The proposed algorithm aims to delete a minimum number of vertices to achieve its goal. The vertex deletion number of a graph $G=(V, E)$ is the smallest integer $k \geq 0$ such that there is an induced planar subgraph of $G$ obtained by the removal of $k$ vertices of $G$. Considering that the corresponding decision problem is NPcomplete and an approximation algorithm for graph planarisation by vertices deletion does not exist, this work proposes an evolutionary algorithm that uses a constructive heuristic algorithm to planarise a graph. This constructive heuristic has time complexity of $O(n+m)$, where $m=|V|$ and $n=|E|$, and it is based on the $P Q$-trees data structure and on the vertex deletion operation. The algorithm performance is verified by means of case studies.
\end{abstract}

\section{INTRODUCTION}

Practical applications of Graph Drawing, such as the design of VLSI circuits, requires drawing techniques for non-planar graphs. A graph (representing the circuit) needs to be drawn on the plane (an electronic chip) without crossing edges. However, graph drawing algorithms are restrained to planar graphs, otherwise the results obtained by these algorithms are compromised. Network design and analysis and computational geometry are additional well known fields where the drawing of planar graphs are required. A possible way to tackle non-planarity in graphs is to consider its topological invariants, such as the number of vertex deletion, which can be used as the measure of non-planarity.

The simple drawing of a graph $G=(V, E)$ is a drawing of $G$ on the plane, where each edge does not cross itself, adjacent edges do not cross themselves, the crossing of two edges only occurs once, the edges do not cross over vertices, and no more than two edges cross at the same point. A graph is considered planar when there is a simple drawing for this graph on the plane, without crossing edges. Without loss of generality, from now on we are considering only simple drawings.
Take all drawings of $G$, the drawing which possesses the lowest number of edge crossings among all drawings is named optimal drawing of $G$. And the number of edge crossings is named crossing number of $G$, denoted by $\operatorname{cr}(G)$.

The number of vertex deletion $\Phi(G)$ is the smallest integer $k>0$ such that the deletion of $k$ vertices from $G$ produces a planar graph. The decision problem regarding the number of vertex deletion, the number of vertex splitting, the number of edges deletion and the crossing number are all NP-complete (Faria et al., 2001a; Garey and Johnson, 1983; Liu and Geldmacher, 1977; Yannakakis, 1978). (Faria et al., 2006) proved that an approximation algorithm cannot exist for the graph planarisation problem using the vertex deletion operation, hence a heuristic algorithm becomes a viable alternative to tackle the problem. Also it has been shown that it remains NP-hard even for cubic graphs (Faria et al., 2001a; Faria et al., 2001b; Faria et al., 2004). Besides, (de Figueiredo et al., 1999) showed that the same occurs for the number of vertices splittings according to the result obtained by (Robertson and Seymour, 1995).

Literature reports many algorithms that attempt to remove a minimal number of edges to obtain a planar subgraph (Chiba et al., 1979; Fisher and Wing, 1966; 
Marek-Sadowska, 1978; Ozawa and Takahashi, 1981; Pasedach, 1976). One of the best approaches is the PLANARISE algorithm by (Jayakumar et al., 1989) referred as JTS PLANARISE algorithm. The JTS PLANARISE algorithm is based on the planarity test algorithm by (Lempel et al., 1967) and (Even, 2011) (also referred as the $L E C$ algorithm) and its implementation using PQ-trees (Booth and Lueker, 1976). (Eades and de Mendonça, 1993) considered the number of vertex splittings adapting the PLANARISE algorithm into the SPLIT-PLANARISE. That was done by replacing the edge removal operation for the vertex splitting operation. Both algorithms have time complexity $O\left(n^{2}\right)$ and space complexity $O(n+m)$, where $n$ represents the number of vertices and $m$ represents the number of edges of $G$. This work proposes an algorithm named VD-PLANARISE which uses similar ideas to the JTS PLANARISE algorithm above mentioned, though it uses the operation of vertex deletion instead of edge removal. In the next section it will be discussed how the JTS PLANARISE algorithm was adapted for the new proposed constructive heuristic which has time and space complexity of $O(n+m)$. We can also highlight that the JTS PLANARISE algorithm generates a planar subgraph where the proposed algorithm generates an induced planar subgraph.

Section 2 describes a few necessary concepts. In section 3 we present the VD-PLANARISE algorithm. The section 4 analyses the complexity and the performance of the proposed algorithm. Section 5 presents the evolutionary algorithm MAVD-PLANARISE and later we show an empirical analysis of the performed tests of the proposed algorithms (section 6).

\section{THE LEC ALGORITHM AND $P Q$-TREES}

This section presents the basics of the JTS PLANARISE algorithm, which is based on the LEC planarity test algorithm which is performed with the aid of $P Q$-trees. The definitions of the data structure and its operations are described in this section. However, for further details on the implementation of the operations on $P Q$-trees we recommend the work of (Booth and Lueker, 1976).

The $L E C$ algorithm only deals with biconnected graphs. Considering that it is fairly easy to divide a graph into a tree of biconnected components (blocks), (Gibbons, 1985) presents a linear complexity algorithm for the generation of a tree of biconnected components for a given graph. This work may consider, thus, only biconnected graphs.

Take a biconnected graph $G=(V, E)$ with $n=|V|$ vertices and $m=|E|$ edges. An $s t$-numbering is a labeling of the vertices in $G$ with integer numbers $1,2, n$ where 1 is adjacent to $n$ and a vertex numbered $j$ is adjacent to a pair of vertices numbered $i$ and $k$ where $i<j<k$. The vertex 1 is named source and is referred as $s$ while the vertex $n$ is named sink and is referred as $t$. Each biconnected graph has a $s t$-numbering (Lempel et al., 1967) and such labeling can be found in linear time (Even and Tarjan, 1976). The graph $G$ labeled with $s t$-numbers is named $s t$ graph.

Let $G_{k}$, where $1 \leq k \leq n$, be a subgraph of an $s t$ graph $G$ induced by the set of vertices $V_{k}=1,2, \ldots, k$. Let $B_{k}$ be a graph associated with the subgraph $G_{k}$ and all of the edges of $G$ connected with the vertices $V_{k}$ and $V-V_{k}$ in $G$. These edges are named virtual edges and the vertices $V-V_{k}$ are named virtual vertices. The virtual vertices are labeled as its original vertices in $G$; though they remain apart (a leaf for each adjacent vertex not yet embedded). Consequently, in $B_{k}$ there may be several virtual vertices with the same label, each of them with exactly one virtual edge. A drawing $B_{k}$ is named bush form of $G_{k}$ if the vertices with smaller or equal labels than $k$ appears at a higher level than the leaves and all of the virtual vertices appears as leaves.

It is possible to demonstrate (Even, 2011; Lempel et al., 1967) that a st-graph is planar if and only if for each $B_{k}, 2 \leq k \leq n-2$, there is a planar graph $B_{k}^{\prime}$ isomorph to $B_{k}$ such that all the virtual vertices in $B_{k}^{\prime}$ labeled $k+1$ appear consecutively.

A $P Q$-tree (Booth and Lueker, 1976) $T$ is a data structure that represents a set of permutations in a set $S$. The nodes of $T$ can be leaves, representing the elements of $S ; P$-nodes, conventionally represented as a circle; and $Q$-nodes, conventionally represented as a rectangle.

For this kind of tree the order that the descendants of a node appear is important. The borderline of $T$ is defined as the permutation represented by the order of the leaves of $T$ from left to right. For example, the borderline of the first $P Q$-tree in Figure 1 is [abcde].

The set of permutations represented by $T$ is generated by rearranging the descendants of each node $P$ and $Q$, according to two rules - the descendants of a $P$-node can be freely permuted and the order of the descendants of a $Q$-node can only be inverted.

The set of permutations of $S$ represented by $T$ is the set of borderlines of the $P Q$-trees obtained from $T$, by rearranging the descendants according to these rules. For example, the set of permutations represented by the $P Q$-tree in Figure 1 is: [abcde], [abced], [cbade], [cbaed], [dabce], [dcbae], [eabcd], [ecbad], $[d e a b c],[d e c b a],[e d a b c]$, and [edcba].

These $P Q$-trees proved to be useful in many prob- 

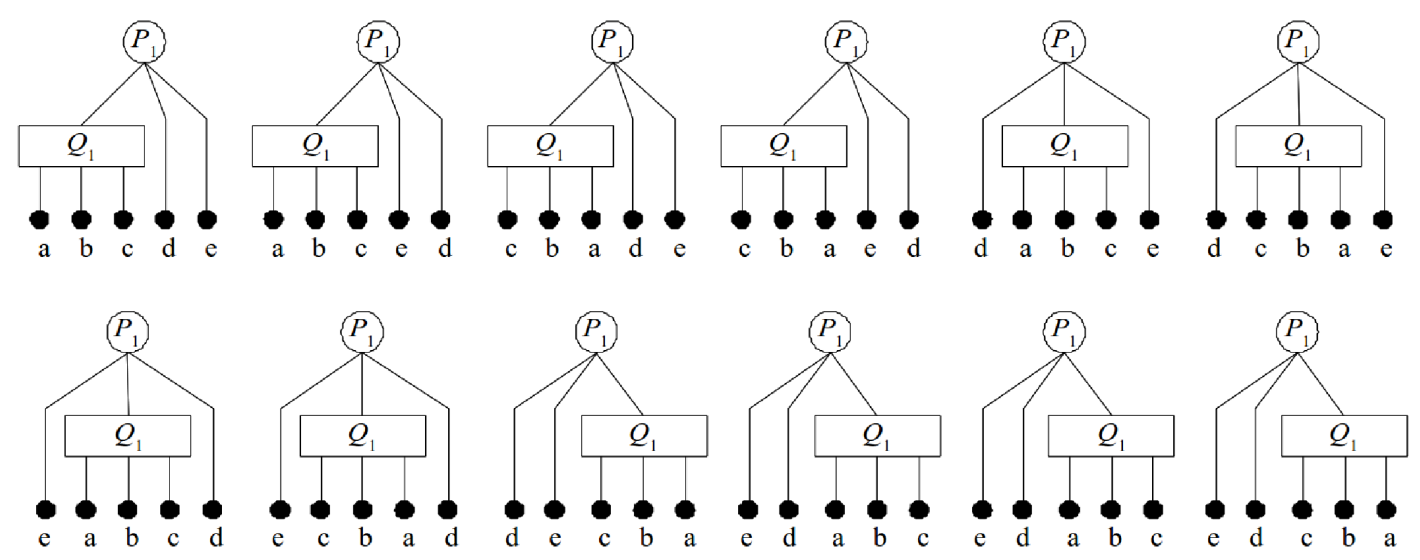

Figure 1: The twelve permutations allowed for the given PQ-tree.

lems involving a successive reduction of the set of permutations to find a specific permutation. For example, they have been used to identify planar graphs, interval graphs, matrix with the property of consecutive ones (Booth and Lueker, 1976), hierarchical planar graphs (Battista and Nardelli, 1988), as well as the dominance drawings (Eades and de Mendonça, 1993).

In this work, a $P Q$-tree $T_{k}$ is used to represent the bush form $B_{k}$ in the algorithm. The nodes of $T_{k}$ correspond to the following:

- leaves: the virtual vertices of $B_{k}$;

- $Q$-nodes: the maximal biconnected components in $B_{k}$; and

- $P$-nodes: the articulation vertices in $B_{k}$.

The leaves are named pertinent if they correspond to the next selected vertices (label $k+1$ ) with the possibility to be embedded, while the others are named non-pertinent leaves. In the same way, a non-leaf node $X$ is pertinent if any leaf descendant of $X$ in the $P Q$-tree is pertinent. If all the leaves from the descendants of a node $X$ in the $P Q$-tree are pertinent, then $X$ is named a full node. If no leaf descendant of the node $X$ is pertinent then $X$ is empty. The $X$ borderline is defined by its set of descendant leaves, read from left to right. A node $X$ is a pertinent root if it is the lowest level node whose borderline has only pertinent leaves. The tree rooted in $X$ is named pertinent subtree. Once a pertinent root is identified, a series of pattern tests and reallocations described in (Booth and Lueker, 1976) can be used in order to build a new tree in which all the pertinent leaves are shown consecutively if such tree exists. In this case all the pertinent leaves in the new tree will appear as descendants of a single node. For instance, suppose that the $P Q$-tree from Figure 2 represents a bush form $B_{7} ; P 1$ node is a pertinent node; $Q 1$ node is an empty node; $P 2$ node is the pertinent root; $Q 2$ node is a full node; the pertinent leaves are labeled as 8 ; and the non-pertinent leaves are labelled as 9,10,11,12.

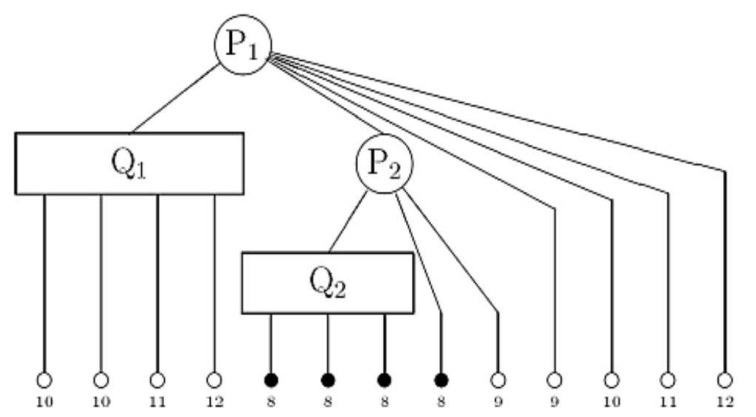

Figure 2: A PQ-tree of a bush form $B_{7}$.

Reduction is an important operation of a $P Q$-tree. On an abstract level, the reduction takes a set of permutations $\Pi$ of $S$ and a subset $S^{\prime} \subseteq S$ and returns a subset $\Pi^{\prime}$ of $\Pi$ in a way that the elements of $S^{\prime}$ consecutively appear in all the permutations in $\Pi^{\prime}$. The elements of $S^{\prime}$ are named pertinent elements of $S$.

(Booth and Lueker, 1976) created an algorithm that reduces a $T_{k}$ tree into a $T_{k}^{*}$ tree in a way that all the pertinent leaves consecutively appear in the borderline (when possible). The reduction operation can be efficiently executed with a sophisticated implementation of $P Q$-trees. This work, however, does not discuss these operations that are detailed in (Booth and Lueker, 1976).

It is trivial to notice that not always a $P Q$-tree $T_{k}$ can be reduced into a $T_{k}^{*}$, thus (Ozawa and Takahashi, 1981) defined some criteria to test a tree before applying the reduction. Let $G$ be a biconnected $s t$-graph and $T_{1}, T_{2}, T_{n-1}$ be the $P Q$-trees corresponding to the bush forms $B_{1}, B_{2}, B_{n-1}$ of $G$. A node $X$ of a $P Q$-tree is classified according to its borderline, as follow:

- Type A: if the rooted subtree in $X$ could be rearranged in a way that all the pertinent leaves de- 
scendant of $X$ consecutively appear in the middle of the borderline, with at least a non-pertinent tree in each extreme of the borderline. For example, the $P_{1}$ node in Figure 2 is the type $A$.

- Type B: if the borderline of the rooted subtree in $X$ consists only of pertinent nodes, then $X$ is a full node. For example, $Q_{2}$ node in Figure 2 is type $B$.

- Type H: if the rooted subtree in $X$ could be rearranged in a way that all the pertinent leaves descendant of $X$ consecutively appear in one of the ends of the borderline. For example, $P_{2}$ node in Figure 2 is type $H$.

- Type $W$ : if the borderline of the rooted subtrees in $X$ consists only of non-pertinent leaves, that is, $X$ is an empty node. For example, $Q_{1}$ node in Figure 2 is type $W$.

It is known that a $P Q$-tree is not always one of the types $A, B, H$ or $W$. However, the need to transform (essentially by vertex deletion) the whole tree in a tree of the $W$ type will be further looked at.

A graph $G$ containing $n$ vertices is planar if and only if the pertinent roots in all the $P Q$-trees, $T_{2}, T_{3}, T_{n-2}$ of $G$ are of the $B, H$ or $A$ type. A $P Q$ tree is reducible if its pertinent root is of $B, H$ or $A$ type, otherwise, it is irreducible (Ozawa and Takahashi, 1981).

Both $T_{1}$ and $T_{2}$ trees are reducible. The first one because it has just a pertinent leaf corresponding to the edge $\left(v_{1}, v_{2}\right)$, and the second one because it has only one type of leaf that is the node corresponding to the virtual vertex $n$.

\section{PLANARISATION BY VERTEX DELETION}

In this section we introduce the proposed graph planarisation constructive heuristic VD-PLANARISE which uses the vertex deletion operation.

In general, the VD-PLANARISE algorithm starts with a vertex and continues with the insertion of one vertex at a time, building an induced planar subgraph $G^{\prime}$ of $G$. The vertices are selected following the labeling order introduced by the $s t$-numbering algorithm (Lempel et al., 1967). Let $v$ be the next candidate vertex to be inserted in the planar subgraph $G^{\prime}$. Let $E_{v}$ be the edge subset incident with the vertex $v$ and the vertices of $G^{\prime}$ and let $\hat{E}_{v}$ be the subset of other edges incident with $v$. For each iteration if a vertex $v$ cannot be inserted into $G^{\prime}$ then it is removed. The remaining set of edges $(v, u) \in \hat{E}_{v}$ is added (as dummy edges) to the first inserted vertex (vertex 1). This will be done to each $u$ vertex that does not have another adjacent with a smaller label comparing to the $v$ label, aiming to maintain the property of $s t$-numbering.

The proposed algorithm is presented as follows:

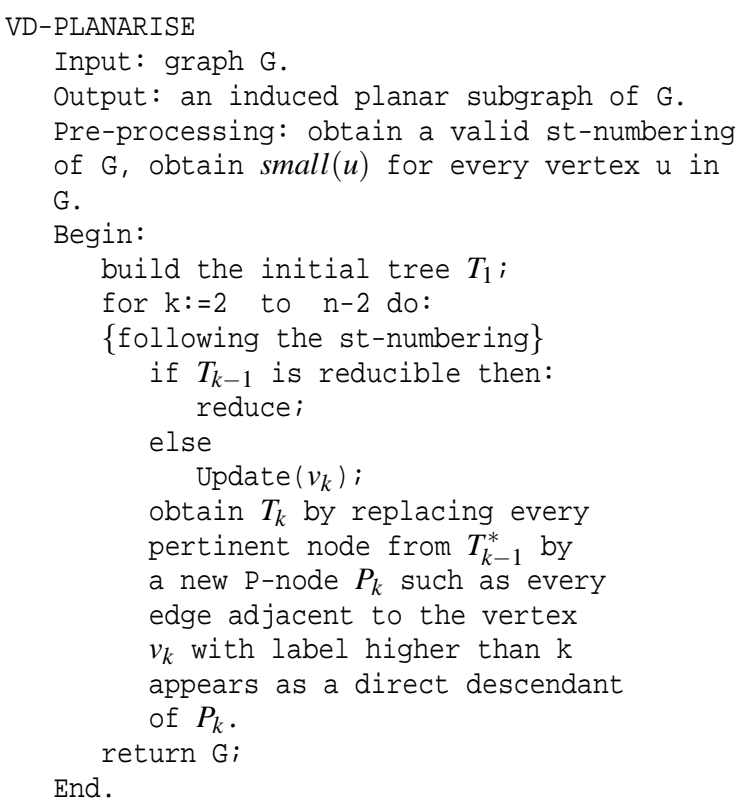

The algorithm starts with the $T_{1}$ tree and builds the sequence of $P Q$-trees $T_{2}, T_{3}$, If a graph is planar the $L E C$ algorithm finishes after building the $T_{n-1}$ tree, otherwise it finishes when it detects the impossibility to reduce a $T_{k}$ tree into $T_{k}^{*}$.

Consider $T_{k}$ an irreducible $P Q$-tree of a non-planar graph, that is, it is impossible to reduce a $T_{k}$ tree into $T_{k}^{*}$. The proposed algorithm adds a new operation named $U p d a t e(k)$. This operation removes all the pertinent leaves transforming the $T_{k}$ tree in type $W$. Besides, if any $u$ vertex with a label higher than $k+1$, adjacent to the equivalent vertex of the removed pertinent leaves does not have any other adjacent vertex with a smaller label, a new edge (dummy) is added to the graph in a way that $u$ is adjacent to $s$. This is necessary to maintain the property of $s t$-numbering. Each immersion iteration of the algorithm can increase the number of the adjacent vertices of $s$. However, this number does not exceed the number of vertices in $G$. The main question is how to inspect the adjacency of the vertices to be removed in order to assure the property of $s t$-numbering without increasing the complexity of time. This can be done by adding a $\operatorname{small}(u)$ field to each vertex $u$. This field informs the amount of $u$ adjacents with smaller labels than the $u$ label established in the step of $s t$-numbering. Thus, when the pertinent nodes -correspondent to the $v_{k+1}$ vertex - are removed to make all the $T_{k}$ subtrees of the $W$ type, the $\operatorname{small}(u)$ field is reduced by one to each adjacent vertex $u$ of $v_{k+1}$ where $u$ has a larger label than $k+1$. 
When the value of the $\operatorname{small}(u)$ field reaches zero, a dummy edge is added from $s$ to $u$. After the last iteration, all the dummy edges from $s$ to $u$ are removed for vertex where $\operatorname{small}(u)$ field is zero.

\section{TIME COMPLEXITY AND PERFORMANCE OF THE VD-PLANARISE}

The Booth and Lueker reduction of all reducible $P Q$ trees $T_{k}$ can be performed in a total time of $O(n+m)$ (Jayakumar et al., 1989). If a $P Q$-tree $T_{k}$ is not reducible, the Update $(k)$ operation that will remove the pertinent vertices is performed. Suppose the worst case with the maximum of removed vertices (notice that it is true for the $K_{n}$ graph). In this case, for each $v$ vertex removed the algorithm inspects the labels of each adjacent vertex $u$ of $v$. If the label of $u$ is larger than the label of $v$, a unit is reduced to the $\operatorname{small}(u)$ value. Thus the Update( $k$ ) operation will inspect each vertex and its adjacents (like BFS algorithm). Hence in the worst case the total time of this operation is $O(n+m)$. The addition of dummy edges to the $s$ vertex is done in the worst case $n$ times. Therefore the complexity of time of VD-PLANARISE is $O(n+m)$.

Since the proposed algorithm is a heuristic one, questions may arise regarding the quality of its solutions, i.e. the amount of vertices removed. The algorithm efficiency - with the exception of a few cases such as the complete graph $K_{n}$ - is highly dependable on the st-numbering, since the $P Q$-trees are built in that order. Hence remains the question: how many different st-numberings can a graph possess? And what is the impact of different $s t$-numberings regarding the quality of the obtained solutions?

It is not trivial to answer these questions since the number of $s t$-numberings of a given graph varies accordingly to its structure and characteristics. For a complete graph consisting of $n$ vertices, after the $s t$ edge is chosen each vertex without a label can be a candidate to receive the next label, thus it is trivial to show that there are $n$ ! possible $s t$-numberings for such graph. It is easy to see that for that case different $s t$ numberings does not affect the solution since every vertex is adjacent to every other vertex, however we can use $n$ ! as an upper bound to the number of possible $s t$-numberings.

Therefore, given the large $s t$-numbering possibilities space and knowing that different $s t$-numbering affects the quality of the solutions obtained by the VD-PLANARISE, we decided to use a search technique to refine the solutions. For additional details regarding the VD-PLANARISE algorithm we recommend the work of (Constantino et al., 2011) and (Pinheiro et al., 2012).

\section{THE EVOLUTIONARY ALGORITHM MAVD-PLANARISE}

As the VD-PLANARISE algorithm possesses linear time complexity, its use as an objective function for optimisation techniques is efficient enough. Knowing that it is possible to run a st-numbering algorithm with linear time complexity and that the stnumberings possibilities space is too large to enumerate, the use of an enhanced mechanism to search a large solution space is viable. Hence we propose the MAVD-PLANARISE.

The objective of the MAVD-PLANARISE is to search for the best parameter setup for both the $s t$ numbering and VD-PLANARISE algorithms. The MAVD-PLANARISE is defined over the basic structure of a memetic algorithm, consisting of a genetic algorithm (Goldberg, 1989) and a local search. The individuals are defined in a structure that contains a copy of the adjacency structure of the graph to be planarised, a $(s, t)$ edge to be used in the st-numbering algorithm, a st vector containing the $s t$-numbering of the graph over that setup and the fitness value (number of removed vertices) calculated after the $s t$ numbering.

The chromosome of an individual is a copy of the adjacency list of the given graph. Let $G$ be a graph and $c$ be a chromosome; $c$ consists of $n$ genes where $n$ is the number of vertices of $G$. Each gene $g_{k}$ is the adjacency list of the vertex $v_{k}$.

Every time an individual is generated - over the initial population generation or by crossover - the $s t$-numbering algorithm is applied over its adjacency structure using the individual selected $(s, t)$ edge with the purpose of obtaining the $s t$-numbering, which is stored in the $s t$ vector. After the $s t$-numbering is obtained, the fitness value is then calculated using the VD-PLANARISE algorithm. Only after that procedure an individual is ready for selection and crossover.

The MAVD-PLANARISE uses a fixed size population with a random initial generation of each individual, copying the adjacency structure of the original graph and randomly swapping the vertices order of each vertex list. During the selection and renewal of the population, we opted for using an elitism system (Goldberg, 1989) of the $10 \%$ best solutions being kept on the population. In order to improve the selection 
chances of less fit individuals and avoid stagnation, the algorithm also utilises a linear scaling (Goldberg, 1989) technique to calculate the fitness value.

Regarding the selection process, after defining the elite, the algorithm uses the roulette method (Goldberg, 1989) to select the pairs for crossover. The selection process using the roulette method uses the fitness value $t_{k}=f_{\text {scaling }}\left(f_{O}(k)\right)$ of each individual of the actual population and the total value $t_{s}=\sum_{k=1}^{n} t_{k}$, where $n$ is the number of individuals of the population. After calculating those values a random value $r$ is picked where $1 \leq r \leq t_{s}$ and the algorithm selects the individuals that belong to the range of the sum of the picked number.

The crossover mechanism of the proposed algorithm is composed by two steps. The first one is a regular uniform crossover operation (Goldberg, 1989) and the second one is what defines the method as a memetic algorithm, a local search to find the best $(s, t)$ edge to be used by the $s t$-numbering algorithm. After the chromosomes are generated and before calculating the fitness, every new individual has a small chance of suffering a mutation. Let this chance be $\alpha$. The mutation process uses the flip technique where one gene is randomly raffled and all the adjacency list of that chosen gene is shuffled.

After the crossover, the MAVD-PLANARISE run a greedy local search procedure for each individual on the neighbourhood of the $(s, t)$ edge with the purpose of choosing the best edge for that graph structure. The procedure begins its search by picking up the best $(s, t)$ edges from the individual parents. The choosing of this edge is made during the crossover and the process consists of finding the best $s t$-numbering given the edge $(s, t)$ and the inverse $(t, s)$ of each parent and the adjacency structure of the generated individual. Only then the greedy search starts by the selected $(s, t)$ edge, its $s t$-numbering and the resulting number of vertices of the graph planarisation using that setup. For each vertex $v$ adjacent to $s$ the GREEDYST$S E A R C H$ algorithm generates a $s t$-numbering using the edge $(s, v)$ as a temporary $(s, t)$ edge and planarise the graph using the VD-PLANARISE algorithm. If the resulting planar subgraph has more vertices than the one with the original $(s, t)$ edge, then the vertex $v$ replaces the vertex $t$ in the $(s, t)$ edge. The greedy search also executes the same procedure for the edge $(v, s)$ and in case the obtained planar subgraph has more vertices than the original one, $(s, t):=(v, s)$ and the GREEDYST-SEARCH algorithm return its recursive call over the new $(s, t)$ edge. The algorithm ends when it cannot find a better solution.

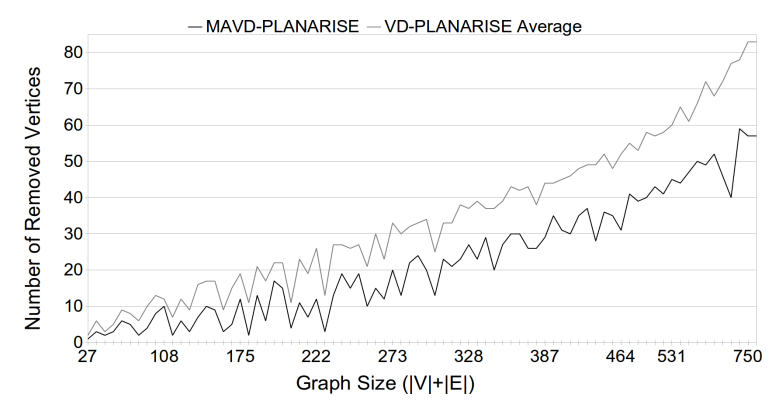

Figure 3: Results for $C_{n} \times C_{m}$ graphs.

\section{RESULTS}

We tested the algorithms on two types of graphs; cartesian graphs, for they possess symmetric and cyclic characteristics among its vertices and edges and randomly generated graphs. For each $n$, where $3 \leq n \leq 10$, we generated ten $C_{n} \times C_{m}$ graphs with $m$ evenly spread through $[n, 25]$, hence obtaining 80 graphs. As for the random graphs, we generated 200 graphs, with $|V|$ evenly distributed such that $30 \leq$ $|V| \leq 75$ and for each pair of vertices we set an edge with a probability of $\delta$, where each graph was given a random $\delta$ value such that $0.25 \leq \delta \leq 0.75$.

As for a measure, we tested the VD-PLANARISE for every possible $s t$-numbering using an enumeration algorithm. The comparative of the quality of the solutions was made between the average and the best solutions obtained by the VD-PLANARISE, and the solution obtained by the MAVD-PLANARISE. Regarding the parametrisation of our proposed memetic algorithm, we defined a population of 100 individuals, and a variable mutation rate $\alpha$ proportional to the population's stagnation, such that $0.05 \leq \alpha \leq 0.15$. For each graph we run the algorithm three times and used the second better result.

Figure 3 presents the chart with the results obtained from the tests on the $C_{n} \times C_{m}$ graphs. The first interesting observation is regarding the discrepancy between the average solutions obtained by the VD-PLANARISE and the solutions found by the MAVD-PLANARISE, meaning that the different st-

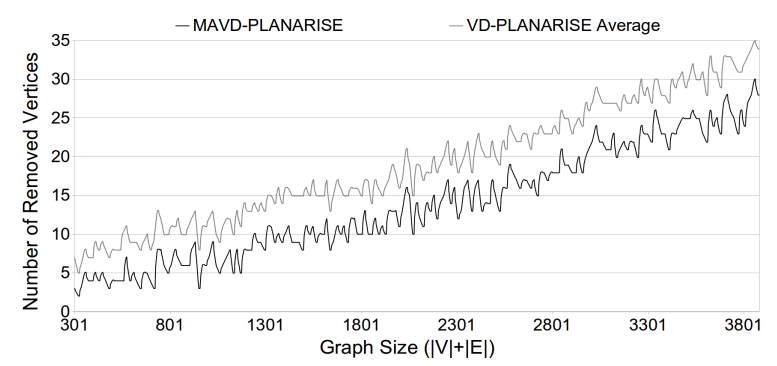

Figure 4: Results for random graphs. 
Table 1: Summary of the experiments.

\begin{tabular}{|c|c|c|c|}
\hline \multicolumn{4}{|c|}{ Cartesian Graphs } \\
\hline \multirow{2}{*}{\begin{tabular}{|l|} 
Total Graphs: \\
Total Vertices: \\
\end{tabular}} & \multicolumn{3}{|c|}{80} \\
\hline & \multicolumn{3}{|c|}{8391} \\
\hline \multirow{2}{*}{$\begin{array}{l}\text { Overall Removed } \\
\text { Vertices: }\end{array}$} & $\begin{array}{l}\text { VD-PLANARISE } \\
\text { Average }\end{array}$ & MAVD-PLANARISE & $\begin{array}{c}\text { VD-PLANARISE } \\
\text { Optimal }\end{array}$ \\
\hline & $\begin{array}{c}2755 \\
32.8 \%\end{array}$ & $\begin{array}{c}1794 \\
21.4 \%\end{array}$ & $\begin{array}{c}1739 \\
20.7 \%\end{array}$ \\
\hline \multirow{5}{*}{$\begin{array}{l}\text { Improvement of the } \\
\text { MAVD-PLANARISE } \\
\text { over the average } \\
\text { solution of the } \\
\text { VD-PLANARISE }\end{array}$} & $\begin{array}{c}\text { Graph Size } \\
(|V+| E \mid)\end{array}$ & $\begin{array}{c}\text { Average } \\
\text { Improvement }\end{array}$ & $\begin{array}{l}\text { Standard } \\
\text { Deviation }\end{array}$ \\
\hline & $<300$ & $47.798 \%$ & $15.246 \%$ \\
\hline & $300-599$ & $30.884 \%$ & $6.666 \%$ \\
\hline & $>600$ & $31.961 \%$ & $8.347 \%$ \\
\hline & Overall & $40.070 \%$ & $14.682 \%$ \\
\hline \multicolumn{4}{|c|}{ Random Graphs } \\
\hline Total Graphs: & \multirow{2}{*}{\multicolumn{3}{|c|}{$\frac{200}{10500}$}} \\
\hline Total Vertices: & & & \\
\hline \multirow{2}{*}{$\begin{array}{l}\text { Overall Removed } \\
\text { Vertices: }\end{array}$} & $\begin{array}{c}\text { VD-PLANARISE } \\
\text { Average }\end{array}$ & MAVD-PLANARISE & $\begin{array}{l}\text { VD-PLANARISE } \\
\text { Optimal }\end{array}$ \\
\hline & $\begin{array}{c}3774 \\
35.9 \%\end{array}$ & $\begin{array}{c}2794 \\
26.6 \%\end{array}$ & $\begin{array}{c}2881 \\
27.4 \%\end{array}$ \\
\hline \multirow{5}{*}{$\begin{array}{l}\text { Improvement of the } \\
\text { MAVD-PLANARISE } \\
\text { over the average } \\
\text { solution of the } \\
\text { VD-PLANARISE }\end{array}$} & $\begin{array}{c}\text { Graph Size } \\
(|V|+|E|)\end{array}$ & $\begin{array}{c}\text { Average } \\
\text { Improvement }\end{array}$ & $\begin{array}{l}\text { Standard } \\
\text { Deviation }\end{array}$ \\
\hline & $<1000$ & $43.252 \%$ & $8.726 \%$ \\
\hline & $1000-1999$ & $29.896 \%$ & $5.695 \%$ \\
\hline & $>2000$ & $21.006 \%$ & $3.595 \%$ \\
\hline & Overall & $31.124 \%$ & $11.091 \%$ \\
\hline
\end{tabular}

numberings in fact have great impact over the quality of the solutions obtained by the planarisation algorithm. Furthermore, we can conclude that for this special class of graphs, the algorithm performs well enough to, in every test case, improve the quality of the obtained solutions.

Figure 4 shows the results obtained from the tests on random graphs. Again, we can observe that the proposed metaheuristic was able to search the solution space and find better solutions.

Table 1 presents a summary of the experiments. For cartesian graphs, we can highlight that the solutions obtained by the MAVD-PLANARISE are very close to the optimal solutions of the VD-PLANARISE, overall only $0.7 \%$ worse, hence proving the quality of the proposed algorithm for this type of graphs. For the random graphs, it can be seen that the performance of the MAVD-PLANARISE is superior to the optimal solution found testing all the st-numberings. This happens because the algorithm not only search in the space of possible $s t$-numberings, but also changes the visiting order of the vertices, which affects directly the $P Q$-trees algorithms and therefore the $V D$ PLANARISE.

The table also presents the mean of the improve-

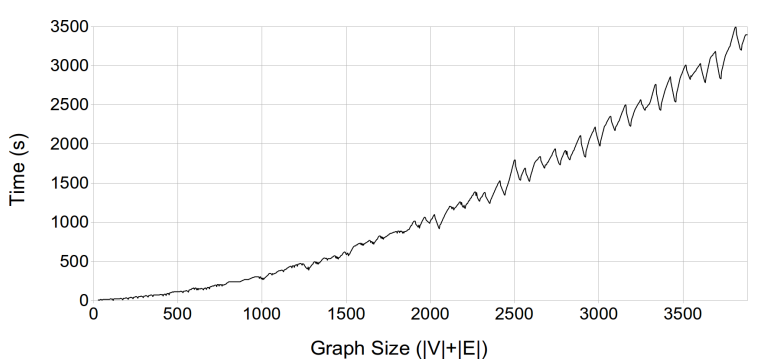

Figure 5: Time curve for the MAVD-PLANARISE. ments achieved by applying the metaheuristic and comparing it to the average solution obtained by the VD-PLANARISE. We can observe that as the size of the graph increases, so the improvement of the solutions obtained by the metaheuristic decreases. This is expected as the search space increases and the problem gets more difficult. Nonetheless, the algorithm performs similar both on cartesian and random graphs as the overall improvements for cartesian graphs was $40.07 \%$ with a standard deviation of $14.682 \%$ and for random graphs with similar size range as the cartesian graphs was $43.252 \%$ with a standard deviation of $8.726 \%$.

The algorithm execution time is shown by the Figure 5 using different sized graphs in terms of number of vertices and edges $(|V|+|E|)$ and the execution time in seconds. It can be noted that the algorithm has a polynomial time performance, with a slightly non-linear growing curve.

\section{CONCLUSION}

This work presented an evolutionary algorithm for graph planarisation - MAVD-PLANARISE - which is based on memetic algorithms. As the planarisation heuristic algorithm, the proposed algorithm applies the VD-PLANARISE which uses the vertex deletion operation to obtain a planar subgraph. To the best of our knowledge this is the only algorithm found in the literature which optimises the number of vertices to be removed for the process of graph planarisation.

It is important to emphasise that in the literature, no linear complexity algorithm that planarises a graph by removing vertices can be found as the use of the vertex deletion operation is not frequent. Note that the proposed algorithm finds an induced planar subgraph from bi-connected non-planar components. However it is possible to find a tree (or a forest in case the graph is not connected) of bi-connected components in linear time complexity and build an induced planar subgraph using successive applications of the algorithm VD-PLANARISE. Hence the algorithm proposed here represents a sound and novel approach to graph planarisation using the vertex deletion.

The proposed MAVD-PLANARISE approach searches the planar solution space using different stnumberings and obtains good results as it was shown in section 6. Although the algorithm is capable of refining the solution, there are no guarantees that by just altering the st-numbering, the VD-PLANARISE can obtain the optimal solution (as expected for a heuristic approach). The MAVD-PLANARISE not only searches in the $s t$-numbering space, but it 
also runs a local search on each individual, further improving the results.

Future research include improvements on the memetic algorithm in order to investigate a wider search space, not just the one provided by the $V D$ PLANARIZE. One option is to use the final solution of the MAVD-PLANARIZE as a starting point to another search procedure (such as simulated annealing, GRASP, VNS, PSO, etc) that does not rely on the VD-PLANARIZE, but instead search in different neighbourhoods. Another option is to adapt such neighbourhoods to the local search procedure already presented in this work. In any case, investigating a wider range of neighbourhoods could potentially improve the quality of the obtained solutions.

\section{REFERENCES}

Battista, G. D. and Nardelli, E. (1988). Hierarchies and planarity theory. IEEE Transactions on Systems, Man, and Cybernetics, (18): 10351046.

Booth, K. S. and Lueker, G. S. (1976). Testing for the consecutive ones property, interval graphs, and graph planarity using pq-tree algorithms. Journal of Computer and System Sciences, 13(3):335 - 379.

Chiba, T., Nishioka, I., and Shirakawa, I. (1979). An algorithm of maximal planarization of graphs. In Proc. 1979 IEEE Symp. on Circuits and Sys, pages 649-652.

Constantino, A. A., de Mendonça, C. F. X., and Pinheiro, R. L. (2011). Um algoritmo heurstico de complexidade linear para planarizaço de grafos por remoço de vrtices. In In Proc. XLIII Simpósio Brasileiro de Pesquisa Operacional, XLIII SBPO, pages 1-11.

de Figueiredo, C. M. H., Faria, L., and Mendonça, C. F. X. (1999). Optimal node-degree bounds for the complexity of nonplanarity parameters. In Proceedings of the tenth annual ACM-SIAM symposium on Discrete algorithms, SODA '99, pages 887-888.

Eades, P. and de Mendonça, C. F. X. (1993). Heuristics for planarization by vertex splitting. In In Proc. ALCOM Int. Workshop on Graph Drawing, GD'93, pages 8385.

Even, S. (2011). Graph Algorithms. Cambridge University Press, New York, NY, USA, 2nd edition.

Even, S. and Tarjan, R. E. (1976). Computing an stnumbering. Theoretical Computer Science, 2(3):339 -344 .

Faria, L., de Figueiredo, C., and Mendonça, C. (2001a). Splitting number is np-complete. Discrete Applied Mathematics, 108(12):65 - 83. ¡ce:title ¿Workshop on Graph Theoretic Concepts in Computer Science;/ce:title

Faria, L., de Figueiredo, C. M. H., and de Mendonça Neto, C. F. X. (2001b). On the complexity of the approximation of nonplanarity parameters for cubic graphs. pages $18-21$.
Faria, L., de Figueiredo, C. M. H., and de Mendonça Neto, C. F. X. (2004). On the complexity of the approximation of nonplanarity parameters for cubic graphs. Discrete Applied Mathematics, 141(1-3):119-134.

Faria, L., de Figueiredo, C. M. H., Gravier, S., de Mendonça, C. F., and Stolfi, J. (2006). On maximum planar induced subgraphs. Discrete Applied Mathematics, 154(13):1774 - 1782.

Fisher, G. and Wing, O. (1966). Computer recognition and extraction of planar graphs from the incidence matrix. Circuit Theory, IEEE Transactions on, 13(2):154163.

Garey, M. and Johnson, D. (1983). Crossing number is npcomplete. SIAM Journal on Algebraic Discrete Methods, 4(3):312-316.

Gibbons, A. (1985). Algorithmic Graph Theory. Cambridge University Press.

Goldberg, D. E. (1989). Genetic Algorithms in Search, Optimization and Machine Learning. Addison-Wesley Longman Publishing Co., Inc., Boston, MA, USA, 1st edition.

Jayakumar, R., Thulasiraman, K., and Swamy, M. (1989). $\mathrm{O}(\mathrm{n} 2)$ algorithms for graph planarization. ComputerAided Design of Integrated Circuits and Systems, IEEE Transactions on, 8(3):257-267.

Lempel, A., Even, S., and Cederbaum, I. (1967). An algorithm for planarity testing of graphs. In Theory of Graphs, International Symposium, pages 215-232.

Liu, P. C. and Geldmacher, R. C. (1977). On the deletion of nonplanar edges of a graph. In Proc. 10th S-E Conf. Combinatorics, Graph Theory, and Computing, Boca, pages 727-738.

Marek-Sadowska, M. (1978). Planarization algorithms for integrated circuits engineering. In in Proc. IEEE International Symposium on Circuits and Systems, pages 919-923.

Ozawa, T. and Takahashi, H. (1981). A graph-planarization algorithm and its applications to random graphs. In in Graph Theory and Algorithms, Lecture Notes in Computer Science, pages 95-107. Springer-Verlag.

Pasedach, K. (1976). Criterion and algorithms for determination of bipartite subgraphs and their application to planarization of graphs. Graphen-Sprach. Algorithm. Graphen, 1. Fachtag. graphen-theor. Konz. Inf., Berlin(West) 1975, 175-183 (1976).

Pinheiro, R. L., Constantino, A. A., and de Mendonça and, C. F. X. (2012). Um algoritmo evolutivo para planarização de grafos por remoção de vértices. In In Proc. XLIV Simpósio Brasileiro de Pesquisa Operacional, XLIV SBPO, pages 1-12.

Robertson, N. and Seymour, P. (1995). Graph minors .xiii. the disjoint paths problem. Journal of Combinatorial Theory, Series B, 63(1):65 - 110 .

Yannakakis, M. (1978). Node-and edge-deletion npcomplete problems. In Proceedings of the tenth annual ACM symposium on Theory of computing, STOC '78, pages 253-264, New York, NY, USA. ACM. 